\title{
Inverse correlation of fluctuations of cerebral blood and water concentrations in humans
}

\author{
Viola Borchardt ${ }^{1, \mathrm{a}}(\mathbb{D})$, Vesa Korhonen ${ }^{1,2}\left(\mathrm{D}\right.$, Heta Helakari ${ }^{1,2}$ (D),

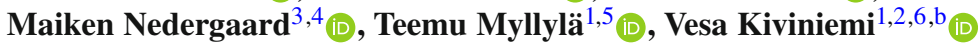 \\ ${ }^{1}$ Oulu Functional Neuroimaging Group, Research Unit of Medical Imaging Physics and Technology (MIPT), \\ University of Oulu, Oulu, Finland \\ 2 Department of Diagnostic Radiology, Medical Research Center (MRC), Oulu University Hospital, Oulu, \\ Finland \\ ${ }^{3}$ Center for Translational Neuromedicine, University of Copenhagen, Copenhagen, Denmark \\ ${ }^{4}$ Center for Basic and Translational Neuroscience, Faculty of Health and Medical Sciences, University of \\ Copenhagen, Copenhagen, Denmark \\ 5 Optoelectronics and Measurement Techniques Research Unit, University of Oulu, Oulu, Finland \\ 6 Present address: Department of Diagnostic Radiology, P.O. Box 50, 90029 Oulu, OYS, Finland
}

Received: 24 October 2020 / Accepted: 22 April 2021

(C) The Author(s) 2021

\begin{abstract}
Near-infrared spectroscopy (fNIRS) measures concentrations of oxygenated $(\mathrm{HbO})$ and deoxygenated ( $\mathrm{HbR})$ hemoglobin in the brain. Recently, we demonstrated its potential also for measuring concentrations of cerebral water $\left(\mathrm{cH}_{2} \mathrm{O}\right)$. We performed fNIRS measurements during rest to study fluctuations in concentrations of $\mathrm{cH}_{2} \mathrm{O}, \mathrm{HbO}$ and $\mathrm{HbR}$ in 33 well-rested healthy control subjects (HC) and 18 acutely sleep-deprived HC. Restingstate fNIRS signal was filtered in full-band, cardiac, respiratory, low-, and very-low-frequency bands. The sum of $\mathrm{HbO}$ and $\mathrm{HbR}$ constitutes the regional cerebral blood volume (CBV). CBV and $\mathrm{cH}_{2} \mathrm{O}$ concentrations were analyzed via temporal correlation and phase synchrony. Fluctuation in concentrations of $\mathrm{cH}_{2} \mathrm{O}$ and $\mathrm{CBV}$ was strongly anti-correlated across all frequency bands in both frontal and parietal cortices. Fluctuation in concentrations of $\mathrm{cH}_{2} \mathrm{O}$ and $\mathrm{CBV}$ showed neither a completely synchronous nor a random phase relationship in both frontal and parietal cortices. Acutely sleep-deprived subjects did not show significant differences in temporal correlation or phase synchrony between fluctuations in $\mathrm{cH}_{2} \mathrm{O}$ and $\mathrm{CBV}$ concentrations compared with well-rested HC. The reciprocal interrelation between fluctuations in CBV and $\mathrm{cH}_{2} \mathrm{O}$ concentrations is consistent with the Munro-Kellie doctrine of constant intracranial volume. This coupling may constitute a functional mechanism underlying glymphatic circulation, which persists despite acutely disturbed sleep patterns.
\end{abstract}

Focus Point on Breakthrough Optics- and Complex Systems-based Technologies of Modulation of Drainage and Clearing Functions of the Brain.

\footnotetext{
a e-mail: borchardt.viola@googlemail.com (corresponding author)

b e-mail: vesa.kiviniemi@oulu.fi
} 


\section{Introduction}

Upon neuronal activation, increases in the oxygenated hemoglobin $(\mathrm{HbO})$ concentration within the blood vasculature are accompanied by reciprocal decreases in deoxygenated hemoglobin (HbR) concentration [1]. This hemodynamic response is the basis for inferences about brain function in several neuroimaging techniques, notably the blood oxygenation level dependent (BOLD) MR signal. An alternative technique called functional near-infrared spectroscopy (fNIRS) is based on light injection and detection on the scalp and reveals fluctuations in both $\mathrm{HbO}$ and $\mathrm{HbR}$ concentrations within a cortical sampling volume [2-5]. The sum of $\mathrm{HbO}$ and $\mathrm{HbR}$ constitutes the regional cerebral blood volume (CBV), which represents a surrogate index for altered neuronal activity in brain tissue [6]. The CBV signal may present a steady time course, but the constituent $\mathrm{HbO}$ and $\mathrm{HbR}$ signals are typically anticorrelated [7], with the direction of change in CBV matching that of $\mathrm{HbO}$. On the other hand, the $\mathrm{HbR}$ concentration is determined by venous blood oxygenation, which is strongly modulated by vasodilation driven by the neuronal tissue [8,9]. Furthermore, cerebral blood flow in gray matter depends on sleep/wake stage, suggesting a mechanism controlling cerebral circulation [10].

Much like blood, cerebrospinal fluid (CSF) comprises $10 \%$ of the volume within the human cranium [11]. CSF consists of $99 \%$ water, is continuously produced in the choroid plexus, and flows through the ventricles and the basal cisterns to the subarachnoid space $[12,13]$. Fluid production at the choroid plexus is thought to create a pressure gradient that determines the direction of net fluid flow, but CSF circulation is also driven by factors such as arterial pulsatility, respiratory venous fluctuations, and slow vasomotion. CSF flow samples the brain interstitial fluid and eliminates macroscopic neurotoxic waste products such as degraded proteins, other metabolites, or electrolytes from the brain [14]. This clearance process is the core function of the intracerebral bulk flow, now known as the "glymphatic system" [15]

In the context of the glymphatic system theory, investigation of CSF dynamics has attracted increasing interest. Obtaining parameters that characterize cerebral fluid flow dynamics and define driving forces of fluid flow in the human brain is a necessary key for understanding glymphatic system function and dysfunction. This need accounts for the emergence of noninvasive approaches to simultaneously measure the concentration changes of cerebral water $\left(\mathrm{cH}_{2} \mathrm{O}\right), \mathrm{HbO}$, and $\mathrm{HbR}$ in humans [16,17].

A recent study by Myllylä et al. demonstrated the possibility to sense dynamic variations of $\mathrm{cH}_{2} \mathrm{O}$ in human using technique based on fNIRS [16]. The changes in brain water signal, measured by fNIRS, are affected by both dynamics of CSF and blood-bound water. As presented in this study, the effect of blood-bound water in $\mathrm{cH}_{2} \mathrm{O}$ signal can be reduced when volume of blood is measured and supposed that concentration changes of blood-bound water in blood vessels are negligible when compared to volume changes in CSF. Unraveling the interrelation of fluctuations in $\mathrm{CBV}$ and $\mathrm{cH}_{2} \mathrm{O}$ concentrations is a fundamental step to gaining better understanding of the mechanisms of fluid dynamics and their interactions in the human brain.

The Munro-Kellie doctrine states that, since the skull is a rigid, confined compartment with a constant intracranial volume, any change in fluid concentration or pressure needs to be counterbalanced to maintain volume equilibrium [18]. In other words, the sum of the volumes of brain matter, CSF, and blood must be constant. A more current and comprehensive view on the Munro-Kellie doctrine includes the whole central nervous system and considers the spinal cord as a viscoelastic extension of the brain. In each cardiac cycle, pulsatile brain deformations driven by physiological pulsations are being relayed on to the ventricles, forc- 
ing CSF displacement from the confined cranium back and forth into the spinal canal [19]. However, due to fundamental reversed anatomical positioning and viscoelastic differences, mean diffusion and convective transport of CSF in gray and white matter are likely to differ between brain and spinal cord [20]. In healthy humans, brain matter is considered to have a fairly constant volume. Although some oscillations in cerebral tissue occur due to cardiovascular and vasomotor pulsations [21], blood and CSF are the major intracranial volume buffers.

Thus, we hypothesized that concentrations of $\mathrm{CBV}$ and $\mathrm{cH}_{2} \mathrm{O}$ would exhibit (1) temporal fluctuations and (2) possibly a counteracting relationship. Furthermore, recent advances have differentiated endogenous brain activity fluctuations that originate from different physiological sources $[21,22]$. We therefore expected that fNIRS measures would exhibit oscillations in the physiological frequency bands, which previously have been shown to drive CSF pulsations. To test these linked hypotheses, we used fNIRS to measure the relationships between fluctuations in hemoglobin and $\mathrm{cH}_{2} \mathrm{O}$ concentrations in 33 well-rested healthy subjects.

Intriguingly, the glymphatic system is mainly disengaged during wakefulness and is activated during sleep or anesthesia [23-26]. Accumulating evidence suggests that sleep disruption interferes with glymphatic clearance and ultimately increases the risk of developing neurodegenerative diseases [24,26-30]. Furthermore, sleep efficiency in patients with cerebrovascular diseases correlates inversely with the volume of the perivascular Virchow-Robin spaces, which are the main channels for glymphatic flow [31]. Given this background, perturbation of normal sleep pattern would affect cerebral fluid interactions in an awake resting state. To test this hypothesis, we studied influences of acute sleep deprivation in 18 healthy subjects after a night without sleep. We supposed that any perturbations in the interrelation of $\mathrm{CBV}$ and $\mathrm{cH}_{2} \mathrm{O}$ concentrations in sleep deprivation states would be more pronounced in well-rested subjects than in acute sleep deprivation.

\section{Methods}

\subsection{Subjects}

Two groups of participants were recruited: 33 well-rested healthy control subjects (HC, 15 females, mean age \pm standard deviation (s.d.): $29.7 \pm 8.1$ years) and 18 healthy subjects performing one night of sleep deprivation (longitudinal design, a subset of the $33 \mathrm{HC}, 8$ females, mean age: $29.7 \pm 6.5$ years). Mann-Whitney $U$ test showed no significant difference in age $(p>0.05)$. Subjects were recruited from the general population by advertisement and screened for absence of neurological disorders, major cardiovascular or respiratory diseases, continuous medication, drug abuse, and shift work. Written informed consent was obtained from each participant prior to testing, in accordance with the Declaration of Helsinki (1983). The regional Ethical Committee of Northern Ostrobothnia Hospital District in Oulu University Hospital approved the study protocols.

\subsection{Experimental design}

Our custom-made MRI-compatible fNIRS using four wavelengths $(660 \mathrm{~nm}, 740 \mathrm{~nm}, 830$ $\mathrm{nm}, 980 \mathrm{~nm}$ ) is presented in [16]. In this device, four NIRS channels were mounted on a DC electroencephalography (EEG) cap with 256 electrodes (manufactured by Electrical Geodesic Incorporation, USA). Two channels were positioned on the left forehead above the eyebrow around the Fp1 electrode (according to the international 10-20 system used for EEG) 
and the other two on the mid parietal cortex near the Pz electrode. The distances between light source and long-range detector and between light source and short-range detector were $3-3.5 \mathrm{~cm}$ and $1-1.5 \mathrm{~cm}$, respectively. Optodes were fixed in place with adhesive tape, and a black spacer protected the eyes from laser reflections.

Subjects assumed a supine position in a 3 Tesla MRI scanner and were asked to lie still, think of nothing specific, and fixate their eyes on a cross displayed on a screen. Hearing was protected via earplugs, and head motion was minimized using soft pads fitted over the ears. For all participants, resting-state fNIRS was measured for $10 \mathrm{~min}$. Well-rested HC were measured in the afternoon. For the sleep-deprivation condition, some of the HC subjects repeated the measurement in the early morning after one night without sleep. During this measurement, the room was not illuminated, and subjects could keep their eyes closed.

\section{3 fNIRS device and measurements}

fNIRS allows the detection of various chemical components of biological tissue based on the absorbance and scattering of light by organic molecules. Light is projected on the tissue, and the back-scattered light at wavelengths ranging from 650 to $1000 \mathrm{~nm}$ can be recorded when using high-sensitive photodetectors for detailed qualitative spectroscopic analysis of chemical structures in the brain. Conventional fNIRS devices usually use two wavelengths to measure blood-flow-related changes in hemoglobin saturation, more precisely $\mathrm{HbO}$ and $\mathrm{HbR}$.

Modifications in this technique enable measurement of other chromophores in the human tissue, for example cytochrome oxidase, fat, or water [32,33]. An isosbestic point corresponds to a specific wavelength at which the absorption spectra of two chromophores cross each other and thus have equal absorbance. To quantify concentrations of two chromophores, it is advisable to select two wavelengths on both sides of the respective isosbestic point, whereas three or more wavelengths are required for the measurement of three chromophores. In this study, we measured $\mathrm{HbO}, \mathrm{HbR}$, and water concentrations. We selected $660 \mathrm{~nm}$ and $830 \mathrm{~nm}$, thus to either side of the isosbestic point $(800 \mathrm{~nm})$ of the $\mathrm{HbO}$ and $\mathrm{HbR}$ absorption spectrum [34]. We chose $980 \mathrm{~nm}$ to probe water activity, since the isosbestic point for water and $\mathrm{HbO}$ is approximately at $930 \mathrm{~nm}[35,36]$. To correct for the respective photosensitivity of each optode, the light intensities were multiplied with the wavelength-dependent photosensitivity.

We note that the measured water signal is potentially comprised of intracellular and extracellular compartments in the measurement volume underneath the optodes. However, intracellular water is not expected to display significant fluctuations in concentration during a 10 -min measurement in a resting subject. Dynamics in the calculated water signal are therefore assumed to be caused by the composite concentration changes in CSF and blood-bound water, here referred to as cerebral water $\left(\mathrm{cH}_{2} \mathrm{O}\right)$.

The additional short-range fNIRS sensor captured mainly dynamics from skin and skull due to its lower penetration depth. Since water and hemoglobin are not thought to cause significant signal fluctuations in skin and skull, the short-range sensor captured extracerebral artifacts and its signal were used to improve quality of the long-range signal [37].

In total, 102 fNIRS datasets were acquired (33 well-rested and 18 acutely sleep-deprived, each in two cortical regions). The raw fNIRS signals were visually inspected to assure quality. In case of a saturated signal or large artifacts, the corrupted part of the signal was removed and only the artifact-free part was used for analysis. The minimum length of signal recordings was $5 \mathrm{~min}$. Four datasets were excluded (two of the well-rested $\mathrm{HC}$ and two of the sleep-deprived group due to malfunctioning frontal laser). After completing two-thirds of the measurements, 
the frontal laser failed and had to be replaced with a laser at $940 \mathrm{~nm}$ that was available at that time.

\section{4 fNIRS data processing}

Raw fNIRS optical intensities were converted into time courses representing concentration fluctuations based on the modified Beer-Lambert law [38-40]. The following extinction coefficients were used: for 660nm: HbR: 3.4408, HbO: 0.3346, H2O: 0.0166; for $830 \mathrm{~nm}$ : HbR: 0.7804, HbO: 1.0507, H2O: 0.1459; for 940 nm: HbR: 0.7874, HbO: 1.3520, H2O: 0.8804; and for $980 \mathrm{~nm}$ : HbR: 0.4233, HbO: 1.2513, H2O: 2.1491 [41]. The differential path length factor was 5.93 [42]. Distances measured between optodes were used in each individual calculation. Calculated mean concentrations for $\mathrm{HbR}, \mathrm{HbO}$, and $\mathrm{cH}_{2} \mathrm{O}$ reflect the total measurement volume underneath the optode.

The time course of signals from the short-range skin detector was subtracted from that of long-range detector to improve detection of signal dynamics in deeper cortical layers [43]. The remaining dynamics in the signals are attributable to physiological events occurring below the skull layer. All signals were separately despiked using interpolation [44], linearly detrended, and low-pass filtered at $0-5 \mathrm{~Hz}$. Total hemoglobin concentration (HbT, equivalent to $\mathrm{CBV}$ representing a surrogate index for neuronal activity) was derived by adding $\mathrm{HbR}$ and $\mathrm{HbO}$ concentrations. To account for differences in signal amplitudes between subjects, the signals were scaled in the range of $[0,1]$. Time courses were additionally band-pass-filtered into four physiological frequency bands, which have been the focus of several previous fNIRS studies [45]: the cardiac band $(0.7-1.5 \mathrm{~Hz})$, the respiratory band $(0.12-0.4 \mathrm{~Hz})$, the range of low-frequency fluctuations $(0.01-0.1 \mathrm{~Hz})$, and very-low-frequency fluctuations $(0.001-0.01$ $\mathrm{Hz})$.

\subsection{Data analysis and statistical analysis}

Frontal and parietal measurements were considered separately because they are independent datasets. All calculations were performed in MATLAB R2017b. fNIRS data from well-rested $\mathrm{HC}$ were used to replicate the previously established relationships between $\mathrm{HbO}, \mathrm{HbR}$, and $\mathrm{CBV}$. To investigate the relationships between hemoglobin and $\mathrm{cH}_{2} \mathrm{O}$, the $\mathrm{CBV}$ signals were pairwise-correlated in time with the $\mathrm{cH}_{2} \mathrm{O}$ signals.

Correlations between fluctuations in $\mathrm{CBV}$ and $\mathrm{cH}_{2} \mathrm{O}$ concentrations were calculated in five frequency bands. Group differences between well-rested and sleep-deprived HC in the temporal relationship between amplitude fluctuations in $\mathrm{cH}_{2} \mathrm{O}$ and $\mathrm{CBV}$ concentrations were calculated using two-sample $t$ tests.

Phase synchrony between $\mathrm{CBV}$ and $\mathrm{cH}_{2} \mathrm{O}$ signals was estimated by calculating the phaselocking value (PLV) based on cardiac, respiratory, and low-frequency bands, because estimation of phase-synchrony requires a filtered signal [46]. PLV is a measure of consistency of phase differences of two signals at a given frequency, where the phase component is obtained separately from the amplitude component. PLV has values in the range of [0 1], where unity represents absolute phase synchrony. A Hilbert transform was used to compute phases of the signals using customized code from the NBT toolbox ([47], RRID: SCR_009612). PLV was calculated in a sliding-window manner with an overlap of $75 \%$ between windows. The length of the windows was adapted to the respective frequency band (window length for cardiac band: $2 \mathrm{~s}$; respiratory band: $10 \mathrm{~s}$; low-frequencies: $120 \mathrm{~s}$ ). For each band, the PLVs were averaged across windows to obtain an optimal compromise between statistical accuracy and stationarity. 
frontal cortex

full band $[0-5 \mathrm{~Hz}]$, corr $=-0.89$
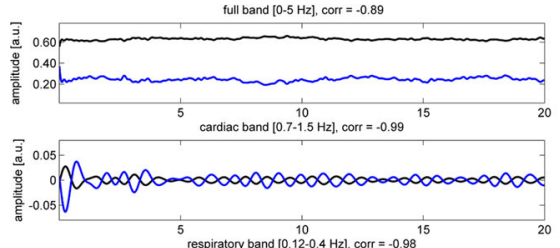

5 respiratory band $[0.12-0.4 \mathrm{~Hz}]$, corr $=-0.98^{15}$

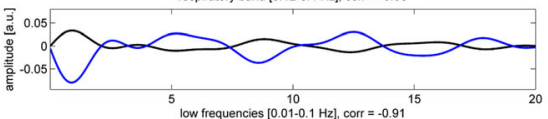

ow frequencies $[0.01-0.1 \mathrm{~Hz}]$, corr $=-0.91$

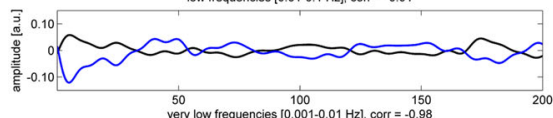

very low frequencies $\left[\begin{array}{ll}100 & 150 \\ {[0.001-0.01 \mathrm{~Hz}] \text {, corr }=} & -0.98\end{array}\right.$

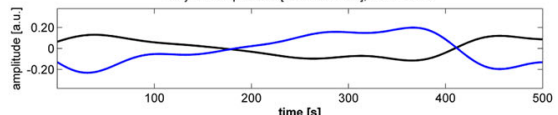

CH2O CBV

parietal cortex
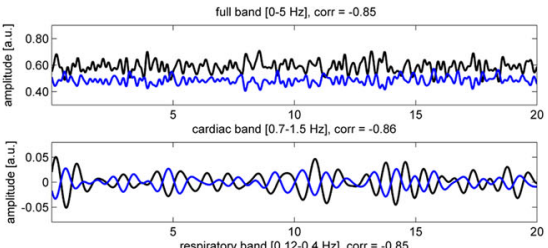

${ }^{5} \stackrel{10}{10} \underset{15}{15}$
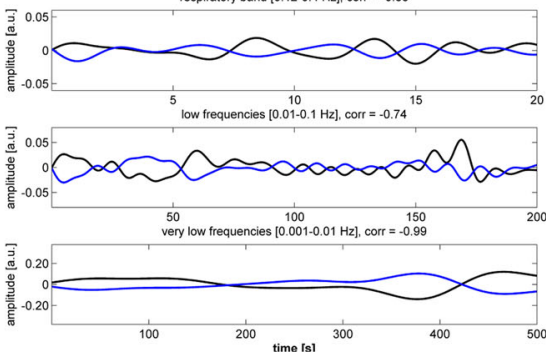

Fig. 1 Filtered fNIRS signals of cerebral water (blue, $\mathrm{cH}_{2} \mathrm{O}$ ) and total cerebral blood volume (black, CBV) concentrations in five frequency bands in both frontal (left panel) and parietal (right panel) cortices depicted for one exemplary well-rested healthy subject. Individual correlation coefficients between temporal fluctuations in $\mathrm{cH}_{2} \mathrm{O}$ and $\mathrm{CBV}$ are given in the title of each subplot

Intra-individual variation was assessed by calculating the absolute difference in correlation coefficients as well as PLV between conditions and paired t-tests were used to compare the intra-individual variations in correlation of $\mathrm{cH}_{2} \mathrm{O}$ and $\mathrm{CBV}$.

\section{Results}

3.1 Relationship between cerebral water and blood volume in well-rested subjects

We initially inspected the signals of $\mathrm{CBV}$ and $\mathrm{cH}_{2} \mathrm{O}$ on a single subject level (Fig. 1). $\mathrm{CBV}$ and $\mathrm{cH}_{2} \mathrm{O}$ concentrations exhibited opposing time courses in both cortical locations and across all frequency bands.

We replicated previously established relationships between $\mathrm{HbO}, \mathrm{HbR}$, and $\mathrm{CBV}$ in the group of well-rested HC subjects (Fig. 2). As expected, fluctuations in $\mathrm{HbO}$ and CBV concentrations were strongly correlated (frontal cortex: mean $=0.88 \pm 0.32$, parietal cortex: mean $=0.79 \pm 0.38$, while the relationship between $\mathrm{HbR}$ and $\mathrm{HbO}$ showed moderate anticorrelations (frontal cortex: mean $=-0.37 \pm 0.51$, parietal cortex: mean $=-0.47 \pm 0.46$ ). Fluctuations in $\mathrm{HbR}$ and $\mathrm{CBV}$ concentration were uncorrelated (frontal cortex: mean = $-0.08 \pm 0.55$, parietal cortex: mean $=-0.06 \pm 0.54$ ). The temporal relationship between amplitude fluctuations in $\mathrm{cH}_{2} \mathrm{O}$ and $\mathrm{CBV}$ concentrations revealed strong anti-correlations (frontal cortex: mean $=-0.76 \pm 0.24$, parietal cortex: mean $=-0.72 \pm 0.28$ ).

The anti-correlations between amplitude fluctuations in $\mathrm{cH}_{2} \mathrm{O}$ and $\mathrm{CBV}$ concentrations were present also in cardiac (frontal cortex: mean $=-0.53 \pm 0.46$, parietal cortex: mean $=$ $-0.76 \pm 0.31$ ), respiratory (frontal cortex: mean $=-0.66 \pm 0.30$, parietal cortex: mean $=$ $-0.75 \pm 0.27$ ), low-frequency (frontal cortex: mean $=-0.77 \pm 0.25$, parietal cortex: mean $=$ $-0.67 \pm 0.39$ ), and very-low-frequency bands (frontal cortex: mean $=-0.80 \pm 0.22$, parietal cortex: mean $=-0.60 \pm 0.43$, Fig. 3 ). 

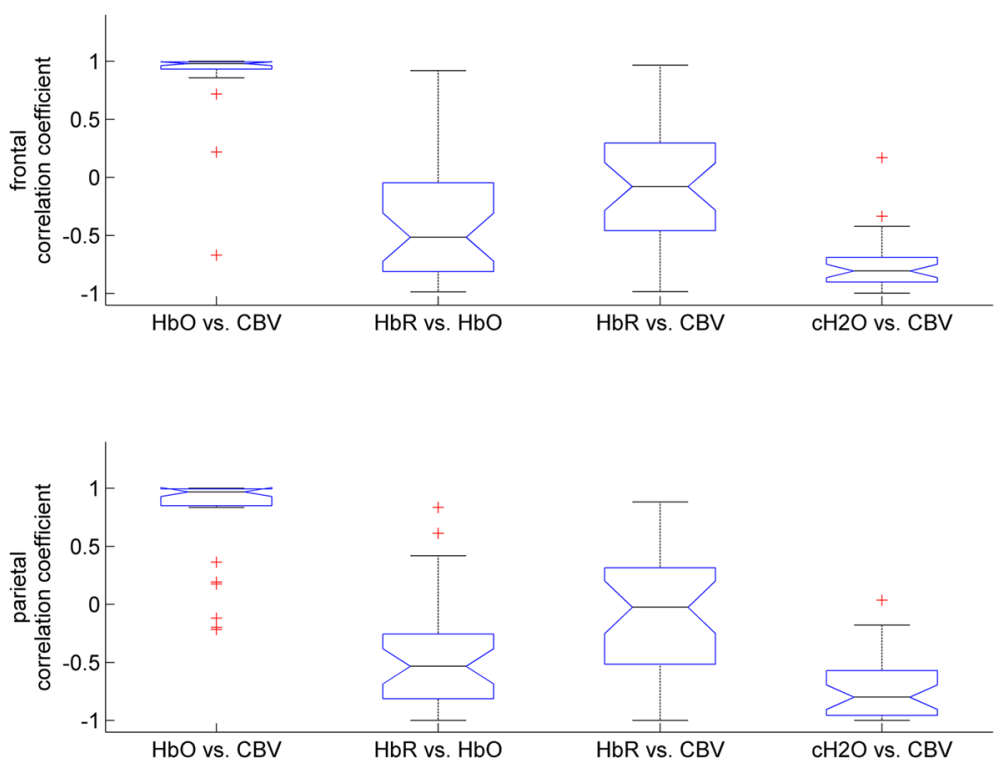

Fig. 2 Correlations between fluctuations in hemoglobin and cerebral water concentrations in both frontal and parietal cortices in the full-band $(0-5 \mathrm{~Hz})$ in healthy well-rested subjects. On each box, the central line indicates the median, and the bottom and top edges indicate the 25 th and 75 th percentiles, respectively. The whiskers extend to the most extreme data points and outliers are plotted as red crosses. HbO: oxygenated hemoglobin, HbR: deoxygenated hemoglobin

Next, we investigated phase synchrony between $\mathrm{CBV}$ and $\mathrm{cH}_{2} \mathrm{O}$ across cardiac, respiratory, and low-frequency bands. $\mathrm{CBV}$ and $\mathrm{cH}_{2} \mathrm{O}$ concentrations had median phase locking values reflecting neither a completely synchronous nor a random relationship and were similar for frontal and parietal cortices (Fig. 4). Phase synchrony between $\mathrm{CBV}$ and $\mathrm{cH}_{2} \mathrm{O}$ concentrations was constant across frequency bands (frontal cortex: cardiac: mean $=0.57 \pm 0.22$, respiratory: mean $=0.58 \pm 0.18$, low frequencies: mean $=0.62 \pm 0.17$; parietal cortex: cardiac: mean $=0.68 \pm 0.20$, respiratory: mean $=0.66 \pm 0.18$, low frequencies: mean $=0.59 \pm 0.24$, Fig. 4).

\subsection{Effect of acute sleep deprivation on relationship between cerebral water and hemoglobin}

To investigate the effect of acute sleep deprivation on the relationship between $\mathrm{cH}_{2} \mathrm{O}$ and $\mathrm{CBV}$ concentrations, individual correlation coefficients were compared between the well-rested and acutely sleep-deprived states.

Also in the sleep-deprived state, the temporal relationship between amplitude fluctuations in $\mathrm{cH}_{2} \mathrm{O}$ and $\mathrm{CBV}$ concentrations revealed anti-correlations (frontal cortex: mean $=-0.57 \pm$ 0.40, parietal cortex: mean $=-0.66 \pm 0.37$, Fig. 5). Group differences between wellrested and sleep-deprived $\mathrm{HC}$ in the temporal relationship between amplitude fluctuations in $\mathrm{cH}_{2} \mathrm{O}$ and $\mathrm{CBV}$ concentrations were not significantly altered (frontal cortex: $p=0.052$, parietal cortex: $p=0.63$ ). Subjects showed variations in their individual direction of change, i.e., increases or decreases, from well-rested to acutely sleep-deprived state. However, a significant effect of acute sleep deprivation in intra-individual variations between $\mathrm{cH}_{2} \mathrm{O}$ and CBV concentrations was not found (all $p>0.05$, Table 1). 

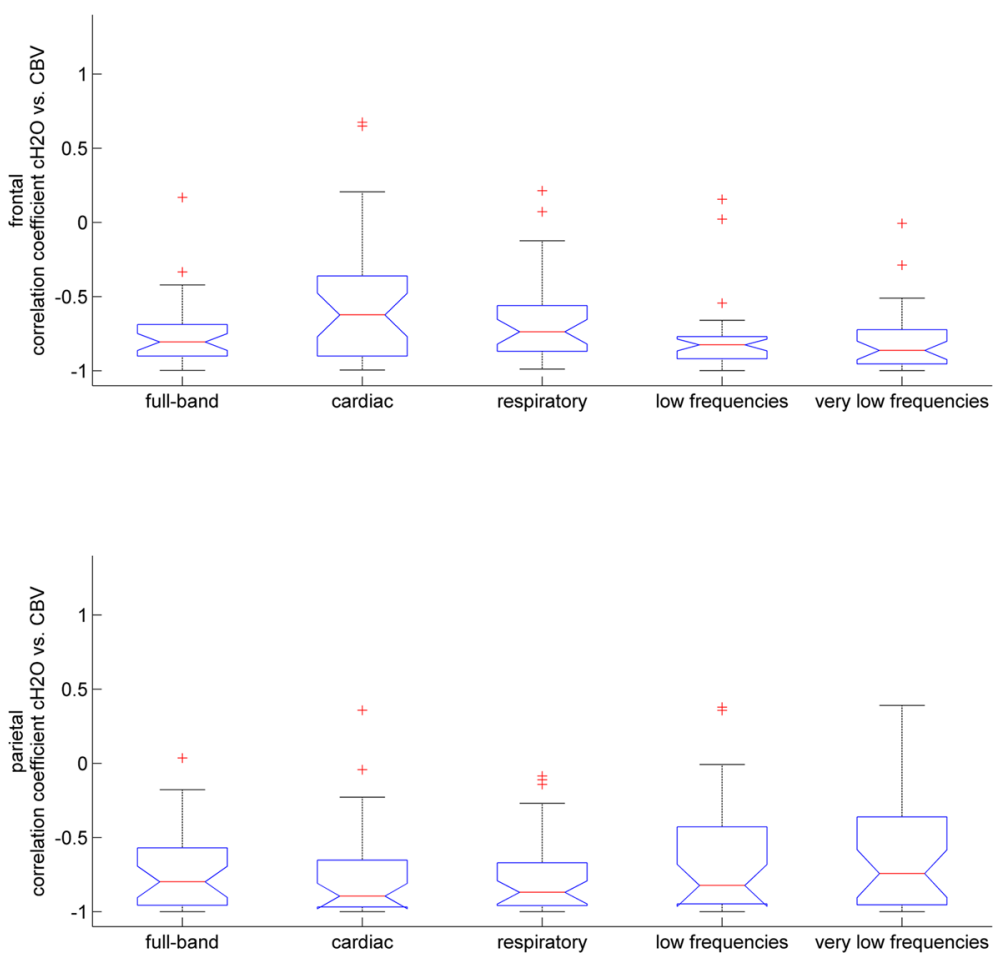

Fig. 3 Correlations between fluctuation in cerebral water $\left(\mathrm{cH}_{2} \mathrm{O}\right)$ and cerebral blood volume (CBV) concentrations in five physiological frequency bands in both frontal (upper panel) and parietal cortices (lower panel) in well-rested healthy subjects. On each box, the central line indicates the median, and the bottom and top edges indicate the 25th and 75th percentiles, respectively. The whiskers extend to the most extreme data points and outliers are plotted as black crosses. $\mathrm{CBV}$ : cerebral blood volume; $\mathrm{cH}_{2} \mathrm{O}$ : cerebral water
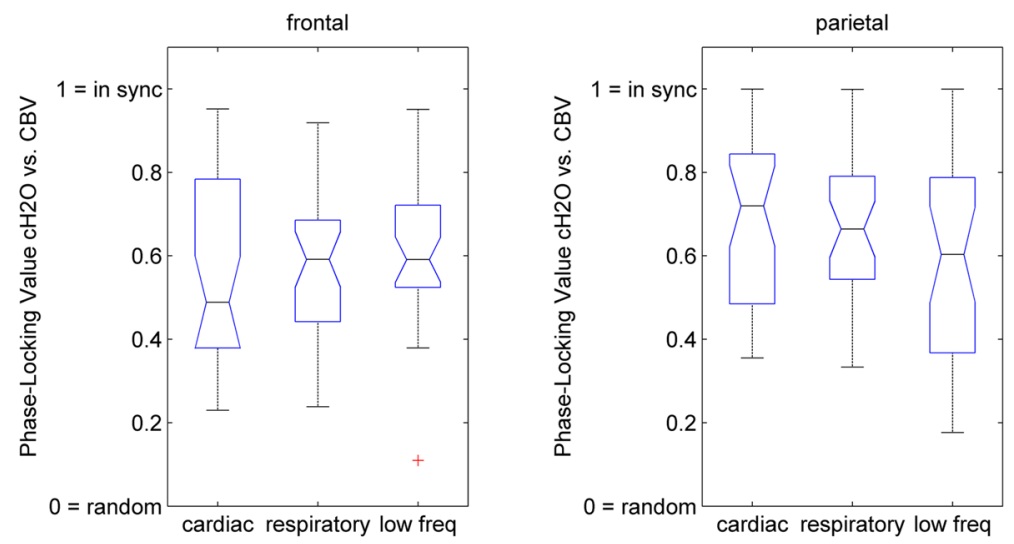

Fig. 4 Phase synchrony of fluctuations between cerebral water $\left(\mathrm{cH}_{2} \mathrm{O}\right)$ and cerebral blood volume (CBV) in three physiological frequency bands (cardiac band $(0.7-1.5 \mathrm{~Hz})$, respiratory band $(0.12-0.4 \mathrm{~Hz})$, low-frequent fluctuations $(0.01-0.1 \mathrm{~Hz})$ ) in both frontal and parietal cortices in well-rested healthy subjects. On each box, the central line indicates the median, and the bottom and top edges indicate the 25th and 75th percentiles, respectively. The whiskers extend to the most extreme data points and outliers are plotted as black crosses 


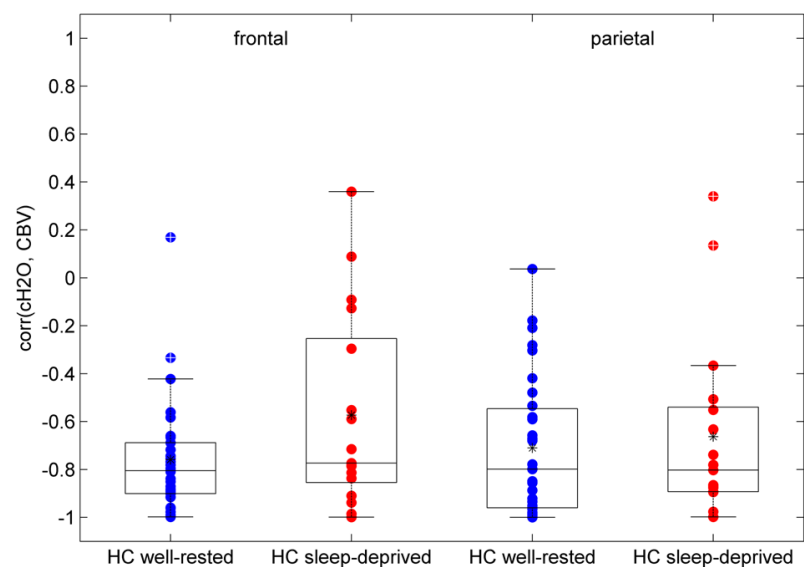

Fig. 5 Effects of acute sleep deprivation on temporal correlation between cerebral water $\left(\mathrm{cH}_{2} \mathrm{O}\right)$ and cerebral blood volume $(\mathrm{CBV})$ in the full frequency band $(0-5 \mathrm{~Hz})$ in both frontal and parietal cortices. Every colored circle represents data from one subject (blue: well-rested subjects, red: acutely sleep-deprived subjects). On each box, the central line indicates the median, the black star the mean, and the bottom and top edges indicate the 25th and 75th percentiles, respectively. The whiskers extend to the most extreme data points and outliers are plotted as white crosses

Table 1 Intra-individual variation expressed as absolute difference $(|\Delta|)$ in correlation coefficient and results of paired $t$ tests between well-rested versus acutely sleep-deprived states in HC subjects in five frequency bands and both frontal and parietal cortices

\begin{tabular}{|c|c|c|c|c|c|}
\hline \multicolumn{6}{|c|}{$\left|\Delta \operatorname{corr}\left(\mathrm{cH}_{2} \mathrm{O}, C B V\right)\right|, p$ value of paired $t$ test } \\
\hline & Full-band & Cardiac & Respiratory & Low frequencies & Very low frequencies \\
\hline Frontal & $5.22, p=0.18$ & $6.70, p=0.39$ & $6.89, p=0.51$ & $4.23, p=0.37$ & $4.64, p=0.30$ \\
\hline Parietal & $4.53, p=0.76$ & $4.54, p=0.38$ & $4.64, p=0.56$ & $7.90, p=0.99$ & $7.51, p=0.037$ \\
\hline
\end{tabular}

Table 2 Intra-individual variation expressed as absolute difference $(|\Delta|)$ in PLV and results of paired $t$ tests between well-rested versus acutely sleep-deprived states in HC subjects in five frequency bands and both frontal and parietal cortices

\begin{tabular}{llll}
\hline$\left|\Delta \mathrm{PLV}\left(\mathrm{cH}_{2} \mathrm{O}, \mathrm{CBV}\right)\right|, p$ value of paired $t$ test & & \\
\hline Cardiac & Respiratory & Low frequencies \\
\hline Parietal & $4.14, p=0.84$ & $3.72, p=0.91$ & $4.37, p=0.50$ \\
& $3.26, p=0.46$ & $2.71, p=0.67$ & $4.29, p=0.88$ \\
\hline
\end{tabular}

Subjects showed individual variations (i.e., increases or decreases) in their PLV between well-rested and acutely sleep-deprived states (Table 2), but no significant effects of acute sleep deprivation on intra-individual variations between $\mathrm{cH}_{2} \mathrm{O}$ and $\mathrm{CBV}$ concentrations were observed across cardiac, respiratory and low-frequency bands (all $p>0.05$, Table 2). 


\section{Discussion}

The presented results are significant in at least two major aspects and in consequence with the previously shown findings presented in [16]. This is the first report of non-invasively measured fluctuations in the concentration of $\mathrm{cH}_{2} \mathrm{O}$ in the human brain, entailing a total of 102 human datasets to provide robust statistical inferences. Second, our inferences on relation between $\mathrm{CBV}$ and $\mathrm{cH}_{2} \mathrm{O}$ were drawn based on two cortical locations, which showed similar patterns. The most interesting finding that fNIRS measurements revealed is that fluctuations in $\mathrm{cH}_{2} \mathrm{O}$ concentration in the human brain were strongly anti-correlated with $\mathrm{CBV}$ across five physiological frequency bands. There was no evidence for phase synchrony between $\mathrm{cH}_{2} \mathrm{O}$ and CBV concentrations, as PLVs consistently fell between absolute synchrony and randomness, which indicates that their time courses were out of phase across the five frequency bands.

Acutely sleep-deprived subjects did not show significant differences in the interrelation of $\mathrm{cH}_{2} \mathrm{O}$ and $\mathrm{CBV}$ when compared to well-rested healthy subjects, during resting-state measurement. However, $\mathrm{HC}$ subjects with acute sleep deprivation showed high intra-individual variability in the direction of change in the correlation, indicating a heterogeneous response to acute sleep deprivation across subjects.

These present observations are difficult to compare to the first presentation of concentration levels of $\mathrm{cH}_{2} \mathrm{O}$ (Figure 7 in [16]), because the subject in that study had been examined in sitting position with the fNIRS channel placed on the occiput. In that study, the fNIRS cerebral water signal showed a positive correlation with the blood oxygen level-dependent (BOLD) signal measured using functional magnetic resonance imaging (fMRI) [16]. There is no previous research utilizing fNIRS to study the interaction of $\mathrm{cH}_{2} \mathrm{O}$ and $\mathrm{CBV}$.

In light of the Munro-Kellie doctrine, we expected an anticorrelation between amplitude fluctuations in the concentration of $\mathrm{CBV}$ and $\mathrm{cH}_{2} \mathrm{O}$, which are together the major buffers in the cranial compartment. The doctrine assumes that the brain matter is incompressible and has a constant volume in healthy humans [18]. If the concentration of one constituent increases in a space of fixed volume, the concentration of another constituent must necessarily decrease to maintain volume equilibrium. Given the long-known anti-correlation between $\mathrm{HbO}$ and $\mathrm{HbR}$ concentrations, one could expect opposing relationships with $\mathrm{cH}_{2} \mathrm{O}$. As known from the fMRI BOLD literature [8], local neuronal activity entails hyperemia and dilation of arteries and veins containing relatively more oxygenated blood. At the same time, concentrations of cerebral water and $\mathrm{HbR}$ are substituted. Upon neuronal deactivation, the venous volume declines, thus increasing the HbR concentration. In this situation, the available space is filled with $\mathrm{CSF}$, thus raising and thereby re-establishing $\mathrm{cH}_{2} \mathrm{O}$. This scenario predicts for an anticorrelation with $\mathrm{cH}_{2} \mathrm{O}$, as depicted schematically in Fig. 6 .

$\mathrm{CBV}$ did not show phase synchrony with $\mathrm{cH}_{2} \mathrm{O}$, which indicates that these signals do not engage in direct oscillatory coupling with a preferred phase difference. This might exclude any functional relevance of their phase relationship. Alternatively, there may be relationships that are not fully uncovered by the measures employed in this study. Relevant information might be hidden in dynamic lag structures, and it is conceivable that dynamic alterations might exist in water exchange between different CSF compartments of the whole central nervous system, i.e., spinal and intracranial spaces [48]. For example, the venous blood compartment has been considered to have a balloon effect during activation-driven increases in blood flow [8]. Also, in contrast to the scull, the venous structures around the spinal canal are not surrounded by bones and may therefore enable a markedly stronger cushioning effect compared to intra-cranial veins. This cushioning effect may well alter the time domain signals measured by NIRS in a nonlinear dynamic way, which can make direct time signal 


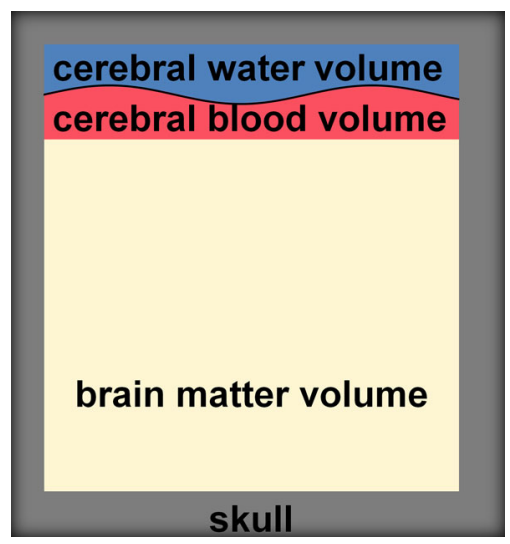

Fig. 6 Schematic representation of the observed relationships in the context of the Munro-Kellie doctrine. The skull frames a confined space, which is $80 \%$ filled with incompressible brain matter, and with $10 \%$ volume fraction for blood and cerebrospinal fluid. If the volume within the skull is constant [18], any change in component volume or concentration is met by a change to maintain equilibrium. Cerebral blood volume (CBV) comprises the sum of oxygenated $(\mathrm{HbO})$ and deoxygenated hemoglobin $(\mathrm{HbR})$ concentrations. CBV and cerebral water volume functionally counteract, which is depicted by the curved line representing opposite fluctuations in concentrations of $\mathrm{CBV}$ and $\mathrm{cH}_{2} \mathrm{O}$ at physiological frequencies measured using non-invasive fNIRS

correlations with constant lag difficult. Furthermore, the spinal canal CSF siphoning of flow is likely to add another source of delay into the correlation analysis. Further multimodal studies to assess these signals during execution of cognitive, affective, or sensory tasks should help resolve this issue.

The observed relationships between $\mathrm{CBV}$ and $\mathrm{cH}_{2} \mathrm{O}$ concentrations were similar in all tested physiological frequency bands, hinting towards the absence of specific influences of any frequency range. Therefore, it seems that the interrelation between $\mathrm{CBV}$ and $\mathrm{cH}_{2} \mathrm{O}$ is not driven by any specific oscillation frequency. This observation is consistent with previous evidence for a contribution of these physiological frequencies to glymphatic pulsation mechanisms [21].

A previous fNIRS study showed that one night of sleep deprivation in healthy subjects reduced the wavelet-based phase coherence between left and right prefrontal oxyhemoglobin oscillations [49]. Another fNIRS study on phase and amplitude of oscillatory components of cerebral hemodynamics during sleep in human subjects showed that blood volume and flow velocity oscillations maintained their relative phase difference during sleep, although their amplitudes were attenuated during non-REM sleep [45]. If sleep disruption indeed altered the coupling pattern in our subjects, we might have expected a higher correlation between fluctuations in $\mathrm{cH}_{2} \mathrm{O}$ and $\mathrm{CBV}$. But the interrelation showed a stable pattern despite acute sleep deprivation, albeit of higher variance. Whether deep sleep alters the observed correlation/anticorrelation patterns remains a question for future research requiring measurements performed also during sleep.

Of note, the novelty of our methodology calls for caution in the interpretation of our results. For example, the analyzed $\mathrm{cH}_{2} \mathrm{O}$ signal may consist of intracellular and extracellular (containing interstitial fluid, CSF, and blood plasma) water compartments in the cortical area underneath the optode. However, these affect mainly the DC level of the measured signals. Thus, shown $\mathrm{cH}_{2} \mathrm{O}$ dynamics are assumed to be caused by concentration changes in CSF, interstitial fluid, and blood-bound water. Due to the current limitations in the spatial 
resolution of fNIRS, it is not possible to localize the exact source of the signal contributing to the $\mathrm{cH}_{2} \mathrm{O}$ concentration. Currently, validation studies for the method are conducted using animal models. For instance, an animal model utilizing thinned skull technique, the similar measurements can be repeated in the absence of light penetrance problems in extracerebral layers.

We emphasize that our present fNIRS methods do not measure whole-brain water content, which are $80-85 \%(w / v)$ in adults [50]. While the brain water content is higher and shows a high variability in infants due to physiological maturation processes and flexibility in the presence of open fontanels, it is nearly constant in adults [51,52]. With fNIRS, the absolute concentration changes in hemoglobin cannot be quantified, and the precise correlation between the fNIRS signal and neural activity remains to be fully understood [2]. These reservations notwithstanding, our present data processing pipeline included removal of artifactual signals from extracerebral tissue as suggested by [37], which greatly improved signal quality and increases the validity of derived inferences. Interregional comparisons of NIRS signal amplitudes are not as reliable as those of hemodynamic changes, because of its vulnerability to spatial variation in skull and subarachnoideal space thickness [53,54].

The present repeated fNIRS measurements in HC subjects had small variations in optode positioning between the two acquisitions, which would result in uncertainty regarding signal localization. However, since the analysis of the relation between $\mathrm{cH}_{2} \mathrm{O}$ and $\mathrm{CBV}$ with high temporal resolution is the main interest of this study, the exact positioning of the optodes is less of an issue.

In this experimental arm of a larger multimodal assessment of brain function, subjects were measured while lying in an MRI scanner. Since a third of subjects likely fall asleep within 3 min of resting-state fMRI studies [55], it is likely that vigilance declined during the measurements in our subjects. Especially for the cases of sleep-deprived HC subjects, we cannot exclude possible interference of light sleep intervals. A detailed evaluation of sleep stages is outside the scope of the current work. Since fNIRS supports essentially unlimited measurement time, future studies may investigate possible effects on vigilance fluctuations on the functional interrelation between $\mathrm{cH}_{2} \mathrm{O}$ and $\mathrm{CBV}$ fluctuations based on 24-hour measurements during normal sleep, sleep deprivation, or sleep restriction, and pharmaco-fNIRS.

The current approach could be applied for clinical research in other patient datasets, for example in Alzheimer's dementia patients, in whom disrupted sleep architecture and glymphatic failure may contribute to neurodegeneration [56]. Indeed, recent glymphatic research showed reduced CSF pressure in patients suffering from Alzheimer's disease [19], which could predict for altered relationships between cerebral water and CBV. Another interesting clinical relationship is intracranial pressure changes occurring in patients with so-called normal pressure hydrocephalus. However, measurement of intracranial pressure is invasive and hence not advisable in healthy volunteers.

Based on the previous proof-of-concept study, we show that fNIRS measurements of $\mathrm{cH}_{2} \mathrm{O}$ and CBV are technically feasible in patients. No study has hitherto supported an analysis of fluctuations in cerebral water concentration. The observed anti-correlation between CBV and $\mathrm{cH}_{2} \mathrm{O}$ may constitute a fundamental functional mechanism underlying glymphatic circulation in the human brain.

Funding Open access funding provided by University of Oulu including Oulu University Hospital. Funding was provided by Academy of Finland (Grant No. 314502).

Open Access This article is licensed under a Creative Commons Attribution 4.0 International License, which permits use, sharing, adaptation, distribution and reproduction in any medium or format, as long as you give appropriate credit to the original author(s) and the source, provide a link to the Creative Commons licence, 
and indicate if changes were made. The images or other third party material in this article are included in the article's Creative Commons licence, unless indicated otherwise in a credit line to the material. If material is not included in the article's Creative Commons licence and your intended use is not permitted by statutory regulation or exceeds the permitted use, you will need to obtain permission directly from the copyright holder. To view a copy of this licence, visit http://creativecommons.org/licenses/by/4.0/.

\section{References}

1. M. Ferrari, V. Quaresima, Neuroimage 63, 921-935 (2012)

2. Y. Hoshi, Philos. Trans. R. Soc. A Math. Phys. Eng. Sci. 369, 4407-4424 (2011)

3. M. Strait, M. Scheutz, Front. Neurosci. 8, 117 (2014)

4. R.C. Mesquita, M.A. Franceschini, D.A. Boas, Biomed. Opt. Express 1, 324-336 (2010)

5. F. Jobsis, Science 198, 1264-1267 (1977)

6. L. Gagnon, R.J. Cooper, M.A. Yücel, K.L. Perdue, D.N. Greve, D.A. Boas, Neuroimage 59, 2518-2528 (2012)

7. A. Kleinschmidt, H. Obrig, M. Requardt, K.-D. Merboldt, U. Dirnagl, A. Villringer, J. Frahm, J. Cereb. Blood Flow Metab. 16, 817-826 (1996)

8. R.B. Buxton, E.C. Wong, L.R. Frank, Magn. Reson. Med. 39, 855-864 (1998)

9. C.N. Hall, C. Reynell, B. Gesslein, N.B. Hamilton, Nature 508, 55-60 (2014)

10. R.E. Townsend, P.N. Prinz, W.D. Obrist, J. Appl. Physiol. 35, 620-625 (1973)

11. A. Thrane, V. Thrane, M. Nedergaard, Trends Neurosci. 36, 52-60 (2009)

12. R. Spector, R.F. Keep, S. Robert Snodgrass, Q.R. Smith, C.E. Johanson, Exp. Neurol. 267, 78-86 (2015)

13. D. Orešković, M. Radoš, M. Klarica, Neuroscience 354, 69-87 (2017)

14. S. Dreha-Kulaczewski, A.A. Joseph, K.-D. Merboldt, H.-C. Ludwig, J. Gartner, J. Frahm, J. Neurosci. 35, 2485-2491 (2015)

15. M. Nedergaard, Science 340, 1529-1530 (2013)

16. T. Myllylä, E. Vihriälä, M. Pedone, V. Korhonen, L. Surazynski, M. Wróbel, A. Zienkiewicz, J. Hakala, H. Sorvoja, J. Lauri, T. Fabritius, M. Jȩdrzejewska-Szczerska, V. Kiviniemi, I. Meglinski, in Proc. SPIE 10063, Dyn. Fluctuations Biomed. Photonics XIV (2017)

17. T. Myllylä, M. Harju, V. Korhonen, A. Bykov, V. Kiviniemi, I. Meglinski, J. Biophotonics 11, e201700123 (2018)

18. B. Mokri, Neurology 56, 1746-1748 (2001)

19. A.A. Linninger, M. Xenos, B. Sweetman, S. Ponkshe, X. Guo, R. Penn, J. Math. Biol. 59, 729-759 (2009)

20. S. Liu, M.A. Lam, A. Sial, S.J. Hemley, L.E. Bilston, M.A. Stoodley, Fluids Barriers CNS 15, 13 (2018)

21. V. Kiviniemi, X. Wang, V. Korhonen, T. Keinanen, T. Tuovinen, J. Autio, P. LeVan, S. Keilholz, Y.-F. Zang, J. Hennig, M. Nedergaard, J. Cereb. Blood Flow Metab. 36, 1033-1045 (2015)

22. J.J. Iliff, H. Lee, M. Yu, T. Feng, J. Logan, M. Nedergaard, H. Benveniste, J. Clin. Investig. 123, 1299-1309 (2013)

23. L. Xie, H. Kang, Q. Xu, M.J. Chen, Y. Liao, M. Thiyagarajan, J.O. Donnell, D.J. Christensen, J.J. Iliff, T. Takano, R. Deane, M. Nedergaard, Science 342, 373-7 (2013)

24. S. Ooms, S. Overeem, K. Besse, M.O. Rikkert, M. Verbeek, J.A.H.R. Claassen, JAMA Neurol. 71, 971977 (2014)

25. E.L. Boespflug, J.J. Iliff, Biol. Psychiatry 83, 328-336 (2017)

26. E. Shokri-Kojori, G.-J. Wang, C.E. Wiers, S.B. Demiral, M. Guo, S.W. Kim, E. Lindgren, V. Ramirez, A. Zehra, C. Freeman, G. Miller, P. Manza, T. Srivastava, S. De Santi, D. Tomasi, H. Benveniste, N.D. Volkow, Proc. Natl. Acad. Sci. 115, 4483-4488 (2018)

27. C.R. Kyrtsos, J.S. Baras, in BIOTECHNO 2014: The Sixth International Conference on Bioinformatics, Biocomputational Systems and Biotechnologies (2014)

28. G. Ringstad, K.-A. Mardal, P.K. Eide, JCI Insight 3, e121537 (2018)

29. R.O. Weller, E. Djuanda, H.-Y. Yow, R.O. Carare, Acta Neuropathol. 117, 1-14 (2008)

30. J.J. Iliff, M. Wang, Y. Liao, B.A. Plogg, W. Peng, G.A. Gundersen, H. Benveniste, G.E. Vates, R. Deane, S.A. Goldman, E.A. Nagelhus, M. Nedergaard, Sci. Transl. Med. 4, 147ra111 (2012)

31. C. Berezuk, J. Ramirez, F. Gao, C.J.M. Scott, M. Huroy, R.H. Swartz, B.J. Murray, S.E. Black, M.I. Boulos, Sleep 38, 853-858 (2015)

32. A. Corlu, R. Choe, T. Durduran, K. Lee, M. Schweiger, S.R. Arridge, E.M.C. Hillman, A.G. Yodh, Appl. Opt. 44, 1948-1956 (2005)

33. V. Tuchin, Tissue Optics: Light Scattering Methods and Instruments for Medical Diagnosis (SPIE Optical Engineering Press, Bellingham, 2007), p. 988 
34. T. Myllylä, V. Korhonen, V. Kiviniemi, V. Tuchin, in Proc. SPIE Optical Techniques in Neurosurgery, Neurophotonics, and Optogenetics II 9305 (2015)

35. M.A. McIntosh, U. Shahani, R.G. Boulton, D.L. McCulloch, Investig. Ophthalmol. Vis. Sci. 51, 2266$2273(2010)$

36. V. Tuchin, Tissue Optics, Light Scattering Methods and Instruments for Medical Diagnostics (SPIE PRESS, Bellingham, 2015)

37. L. Gagnon, M.A. Yücel, M. Dehaes, R.J. Cooper, K.L. Perdue, J. Selb, T.J. Huppert, R.D. Hoge, D.A. Boas, Neuroimage 59, 3128-3138 (2012)

38. M. Cope, Near-Infrared Spectroscopy, Applied Aspects of Ultrasonography in Humans (InTech, London, 2012), p. 190

39. D.A. Boas, T. Gaudette, G. Strangman, X. Cheng, J.J.A. Marota, J.B. Mandeville, Neuroimage 13, 76-90 (2001)

40. G.E. Strangman, V. Ivkovic, Q. Zhang, J. Appl. Physiol. Japn. 2, 124 (2017)

41. P. Van der Zee, M. Cope, S. Arridge, M. Essenpreis, L. Potter, A. Edwards, J. Wyatt, D. McCormick, S. Roth, E. Reynolds, Adv. Exp. Med. Biol. 316, 143-153 (1992)

42. M. Cope, The application of near infrared spectroscopy to non invasive monitoring of cerebral oxygenation in the newborn infant. Ph.D. thesis (1991)

43. L. Gagnon, M.A. Yücel, D.A. Boas, R.J. Cooper, Neuroimage 85, 6-27 (2014)

44. N. Mori, T. Suzuki, S. Kakuno, J. Eng. Mech. 133, 122-127 (2007)

45. M.L. Pierro, A. Sassaroli, P.R. Bergethon, B.L. Ehrenberg, S. Fantini, Neuroimage 63, 1571-1584 (2012)

46. F. Varela, J. Lachaux, E. Rodriguez, J. Martinerie, Nat. Rev. Neurosci. 2, 229-239 (2001)

47. S.-S. Poil, Neurophysiological Biomarkers of Cognitive Decline: From Criticality to Toolbox. Ph.D. thesis, Free University of Amsterdam (2013)

48. M.A. Lam, S.J. Hemley, E. Najafi, N.G.F. Vella, L.E. Bilston, M.A. Stoodley, Sci. Rep. 7, 12924 (2017)

49. L. Bu, M. Zhang, J. Li, F. Li, H. Liu, Z. Li, PLoS ONE 12, e0177321 (2017)

50. J. Dobbing, J. Sands, Arch. Dis. Child. 48, 757-767 (1973)

51. M. Wolf, G. Naulaers, F. Van Bel, S. Kleiser, G. Greisen, J. Near Infrared Spectrosc. 20, 43-55 (2012)

52. A. Demel, M. Wolf, C.F. Poets, A.R. Franz, BMC Pediatr. 14, 1-6 (2014)

53. S. Wang, N. Shibahara, D. Kuramashi, S. Okawa, N. Kakuta, E. Okada, A. Maki, Y. Yamada, Opt. Rev. 17, $410(2010)$

54. Y. Hoshi, Progress in Brain Research: Hemodynamic Signals in FNIRS (Elsevier, Amsterdam, 2016), pp. 153-179

55. E. Tagliazucchi, H. Laufs, Neuron 82, 695-708 (2014)

56. H. Benveniste, X. Liu, S. Koundal, S. Sanggaard, H. Lee, J. Wardlaw, Gerontology 06519, 1-14 (2018) 